Supporting Information for

\title{
Near Infrared Electrogenerated Chemiluminescence from Aqueous Soluble Lipoic Acid Au Nanoclusters
}

\author{
Tanyu Wang, ${ }^{\text {a }}$ Dengchao Wang, ${ }^{\mathrm{b}}$ Jonathan W. Padelford, ${ }^{\mathrm{a}}$ Jie Jiang ${ }^{\mathrm{c}}$ and Gangli \\ Wang ${ }^{\mathrm{a} *}$ \\ ${ }^{a}$ Department of Chemistry, Georgia State University, Atlanta, Georgia 30302, United States \\ ${ }^{b}$ Present address: Department of Chemistry and Biochemistry, Queens College-CUNY, Flushing, New York 11367, United States. \\ ${ }^{c}$ Present address: PharmaCenter Bonn, Pharmaceutical Institute, Pharmaceutical Chemistry I, University of Bonn, An der \\ Immenburg 4, D-53121 Bonn, Germany \\ * Tel: 1-404-413-5507; Fax: 1-404-413-5505; Email: glwang@gsu.edu
}

List:

Synthesis and measurement details

Scheme S1. Molecular structures of DEDA-LA on $\mathrm{Au}_{22} \mathrm{NCs}$.

Figure S1. Cyclic voltammograms of (A) Au-LA NCs and (B) DEDA.

Figure S2. ${ }^{1} \mathrm{H}$ NMR spectra of (A) Au-LA and (B) Au-LA-DEDA NCs in $\mathrm{D}_{2} \mathrm{O}$.

Figure S3. UV-vis (A) and photoluminescence (B) spectra of Au-LA and Au-LA-DEDA.

Figure S4. ECL of Au-LA-DEDA NCs with different long-pass filters. 


\section{Synthesis and Characterization of Au-LA-DEDA clusters}

\section{Materials}

Tetrachloroauric acid trihydrate $\left(\mathrm{HAuCl}_{4} \cdot 3 \mathrm{H}_{2} \mathrm{O},>99.99 \%\right)$, lipoic acid (LA, $\geq 99 \%$ ), sodium borohydride $\left(\mathrm{NaBH}_{4}, \geq 99 \%\right)$, sodium hydroxide $(\mathrm{NaOH}, \geq 97 \%)$, deuterium oxide $\left(\mathrm{D}_{2} \mathrm{O}, \geq 99.9 \%\right), \quad N, N$-Diethylethylenediamine (DEDA, $\left.\geq 99 \%\right), \quad N-\left(3^{-}\right.$ dimethylaminopropyl)- $N$ '-ethylcarbodiimide hydrochloride (EDC), sodium phosphate monobasic $\left(\mathrm{NaH}_{2} \mathrm{PO}_{4}, \geq 99.5 \%\right)$ and sodium phosphate dibasic $\left(\mathrm{Na}_{2} \mathrm{HPO}_{4}, \geq 99.0 \%\right)$ were purchased from Sigma-Aldrich and used as received. Phosphate buffer solution (o.2 M) was prepared by mixing different volumes of $\mathrm{Na}_{2} \mathrm{HPO}_{4}$ (o.2 M) and $\mathrm{NaH}_{2} \mathrm{PO}_{4}$ $(0.2 \mathrm{M})$ solution. In all preparations, nanopure water $(>18 \mathrm{M} \Omega \cdot \mathrm{cm})$ from a Barnstead system was used.

\section{Synthesis of Au Nanoclusters Stabilized by Lipoic Acid}

The synthesis followed reported procedure with some modifications. ${ }^{1,2}$ Lipoic acid (o.15 mmol) was first dissolved in nanopure water at slightly basic $\mathrm{pH}$ adjusted with $\mathrm{NaOH}$ for better solubility. A $\mathrm{HAuCl}_{4}$ solution was then added to reach a final Au:LA mole ratio of 1:3 under vigorous stirring. The final solution $\mathrm{pH}$ was adjusted to 11.0. The $\mathrm{Au}(\mathrm{III})$ - disulfide reaction was stirred over ca. $4 \mathrm{~h}$. After the completion of absorbance transitions to largely colorless, $\mathrm{NaBH}_{4}$ (2 equiv) was added at either room temperature or heated at $65{ }^{\circ} \mathrm{C}$. The reaction proceeded over a period of time determined by changes in luminescence. The crude products were filtered to remove any insoluble precipitates, concentrated by removal of solvent via evaporator, and followed by purification. The synthesized NCs were subject to dialysis using regenerated cellulose dialysis tube in nanopure water, which was recharged every few hours over a period of 3- 4 days. The final products were collected after the removal of solvent under vacuum at room temperature. The partial oxidation of sulfur atoms and the alkane chain of the LA (ref. 33) add additional challenges to obtain single crystals. While a distribution from the average composition of $\mathrm{Au}_{22} \mathrm{LA}_{12}$ is expected, other distinct sizes are not anticipated from the absorption spectrum ( $\left.\mathrm{FigS}_{3}\right)$.

\section{Synthesis of Au-LA-DEDA by coupling reactions}

The purified Au-LA NCs were dissolved in 0.2 M PBS at pH 6.o. EDC (20-fold molar excess of EDC to particles) was then directly added into the $\mathrm{Au}_{22} \mathrm{LA}_{12}$ solution. The components were mixed under stirring and reacted for $20 \mathrm{~min}$ at room temperature. The solution $\mathrm{pH}$ was adjusted to 7.2 to quench the activation reaction followed by the addition of DEDA, resulting in a 20 -fold molar excess of amine to particles. The reaction proceeded for $4 \mathrm{~h}$ at room temperature under stirring. The solution $\mathrm{pH}$ was increased to 8.5 to quench the coupling reaction. The coupled NCs were ultracentrifuged thrice at $12000 \mathrm{rpm}$ for 30 min with filter membranes of ${ }_{3} \mathrm{~K}$ molecular weight cutoff. The final products were collected after the removal of solvent under vacuum at room temperature. ${ }^{1} \mathrm{H}$ NMR was recorded for the same sample before and after coupling with DEDA in $\mathrm{D}_{2} \mathrm{O}$.

\section{Characterizations and ECL Measurements}

UV-visible spectra were recorded using a Shimadzu UV-17oo spectrophotometer. Fluorescence spectra were measured with a Horiba Jobin-Yvon Fluorolog 311 spectrometer equipped with PMT visible and InGaAs near IR detectors. A Hoya Y-44 
440 nm-long-pass filter was used to collect emission spectra. ${ }^{1} \mathrm{H}$ NMR spectra were recorded with a Bruker NMR $400 \mathrm{MHz}$ spectrometer.

A three-electrode system in $1 \mathrm{~mm} \times 10 \mathrm{~mm}$ quartz cuvette was used. A Pt mesh was inserted in the light path of $1 \mathrm{~mm}$ as working electrode. $\mathrm{An} \mathrm{Ag} / \mathrm{AgCl}$ wire as quasireference electrode and a $\mathrm{Pt}$ foil as counter electrode were used. The supporting electrolyte was o.1 $\mathrm{M} \mathrm{NaClO}_{4}$. The emission intensity was recorded with an Andor iDUS CCD camera (Model DU401A-BR-DD) under ambient conditions without purging. The working electrode and the cuvette are aligned at a fixed position with respect to the camera for consistency.

Scheme S1. Molecular structures of DEDA-LA on $\mathrm{Au}_{22} \mathrm{NCs}$. The average composition is $\mathrm{Au}_{22}-\mathrm{LA}_{5}-(\mathrm{LA}-\mathrm{DEDA})_{7}$. In other words, there are five carboxylic acid groups remain unmodified by DEDA on average.
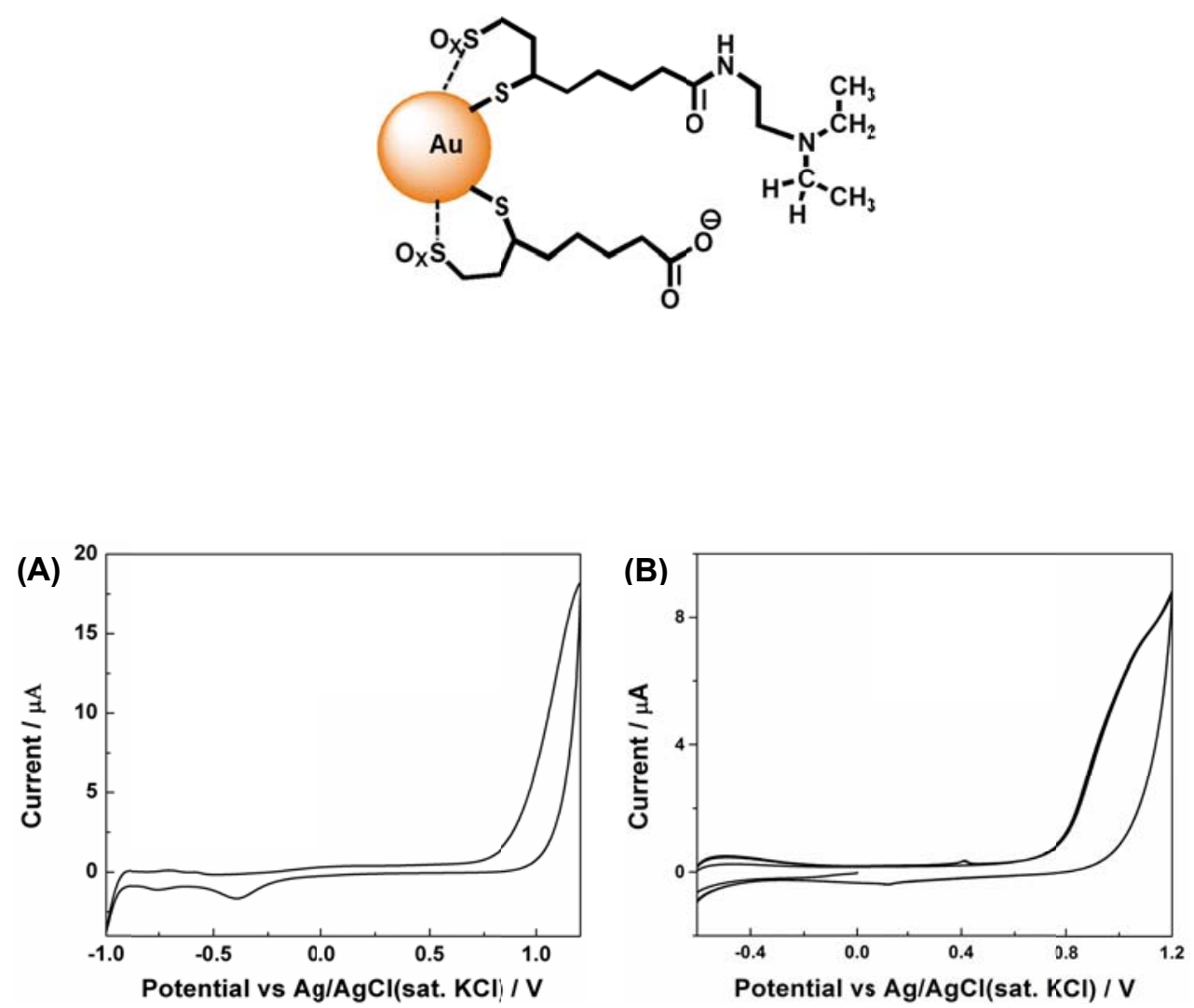

Figure S1. Cyclic voltammograms of (A) Au-LA NCs and (B) DEDA. Both in o.1 M $\mathrm{NaClO}_{4}$ at a scan rate of $0.1 \mathrm{~V} / \mathrm{s}$. 


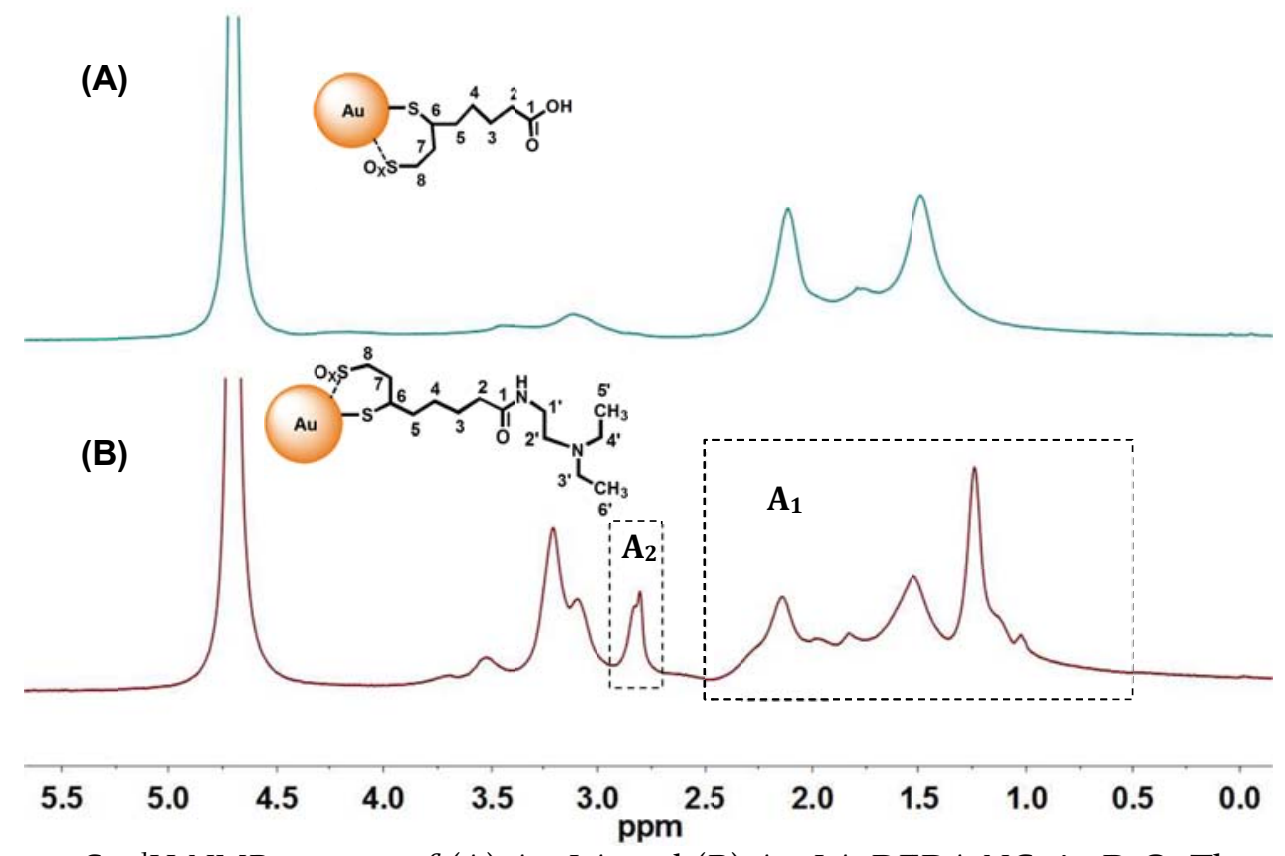

Figure S2. ${ }^{1} \mathrm{H}$ NMR spectra of (A) Au-LA and (B) Au-LA-DEDA NCs in $\mathrm{D}_{2} \mathrm{O}$. The peak assignments in the ${ }^{1} \mathrm{H}$ NMR spectrum for Au-LA NCs are consistent with our previous reports. ${ }^{1}$ After coupling with DEDA, the peak from 1.0 to $1.25 \mathrm{ppm}$ include the methyl protons on C-5' and C- 6 ' while the peaks at 2.8 and $3.2 \mathrm{ppm}$ are assigned to the protons on C-1' and C-2', C-3', C-4' respectively. The number of DEDA coupled on Au-LA NCs was calculated as follow: 1) integration of the peak area from 0.5 to $2.5 \mathrm{ppm}\left(\mathrm{A}_{1}\right)$ and the peak area from 2.7 to $2.9 \mathrm{ppm}\left(\mathrm{A}_{2}\right)$; $\mathrm{A}_{1}$ corresponding to 10 protons on $\mathrm{C}-2, \mathrm{C}-3, \mathrm{C}-4$, $C-5$, and $C-7$ and 6 protons on $C-5$ ' and $C-6$ ' while $A_{2}$ corresponding to 2 protons on $C-$ 1 ; 2) The ratio of DEDA to LA is calculated based on the equation $\left(A_{2} / 2\right):\left(A_{1}-A_{2} \times 3\right) / 10$. Accordingly, there are 7 DEDAs coupled on the LA ligands per Au-LA-DEDA NCs, with 5 LA ligands remaining unmodified.
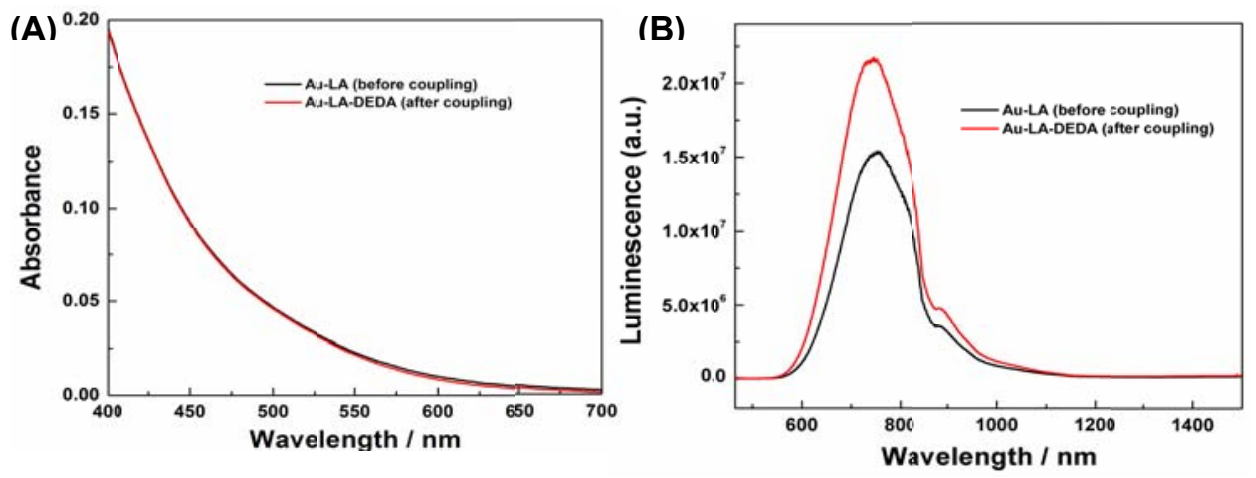

Figure S3. UV-vis (A) and photoluminescence (B) spectra of Au-LA (black curve) and Au-LA-DEDA (red curve) in $\mathrm{H}_{2} \mathrm{O}$. The increase of fluorescence intensity upon coupling is consistent with previous results, due to a decrease of the terminal COO-groups and the introduction of possible positive charges at the amine terminal group as described in the literature. ${ }^{3,4}$ The large Stokes shift is consistent with literature of 
photoluminescence from various $\mathrm{Au}$ nanoclusters and reflects the energy relaxation involved in the core-ligand charge transfer.

(A)

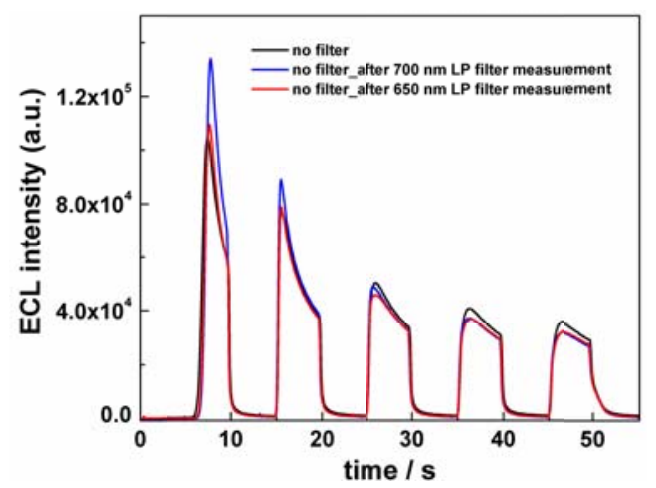

(B)

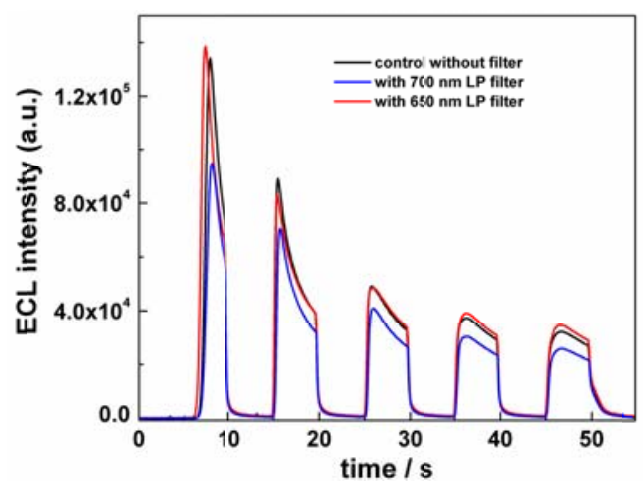

Figure S4. ECL profiles of Au-LA-DEDA NCs without filters as controls (A), and ECL intensity of Au-LA-DEDA NCs with $650 \mathrm{~nm}$ and $700 \mathrm{~nm}$ long-pass filters (B) recorded in $0.1 \mathrm{M} \mathrm{NaClO}_{4}$ under ambient conditions without purging. The integrated intensity of the second peak was analyzed as discussed in the main text. The numbers are consistent with the average of all four peaks (excluding the first one). Note the three controls have the same intensity (within errors) prior to and after the two measurements with filters, attesting the sample stability and negligible surface fouling of the electrode.

\section{References:}

(1) Jiang, J.; Conroy, C. V.; Kyetny, M. M.; Lake, G. J.; Padelford, J. W.; Ahuja, T.; Wang, G. L. J. Phys. Chem. C 2014, 118, 20680.

(2) Shang, L.; Azadfar, N.; Stockmar, F.; Send, W.; Trouillet, V.; Bruns, M.; Gerthsen, D.; Nienhaus, G. U. Small 2011, 7, 2614.

(3) Wu, Z. K.; Jin, R. C. Nano Lett. 2010, 10, 2568.

(4) Wang, G. L.; Huang, T.; Murray, R. W.; Menard, L.; Nuzzo, R. G. J. Am. Chem. Soc. 2005, 127, 812. 